\title{
Application of Near-Infrared Spectroscopy for the Evaluation of Placental Oxygenation
}

\author{
Jun Kakogawa ${ }^{1}$ and Naohiro Kanayama ${ }^{2}{ }^{*}$ \\ ${ }^{I}$ Department of Obstetrics and Gynecology, National Center for Global Health and Medicine, Tokyo, Japan \\ ${ }^{2}$ Department of Obstetrics and Gynecology, Hamamatsu University School of Medicine, Hamamatsu, Japan
}

\begin{abstract}
Aims: As the utero-placental circulation have major impact on the fetus, and the placental function can be critical for determining fetal conditions, the placental oxygenation status was investigated.

Methods: Near-infrared spectroscopy (NIRS) is a non-invasive technique used for the evaluation of regional tissue oxygenation in a number of organs. The trans-abdominal NIRS was developed as a non-invasive technique for monitoring placental oxygenation, which was evaluated by the ratio of oxygenated hemoglobin to total hemoglobin (\%) (tissue oxygenation index ,TOI) values. The near-infrared light was confirmed to reach the placenta using the NIRS probe with an inter-optode distance of $4.5 \mathrm{~cm}$ and the NIRO apparatus (Hamamatsu Photonics, Hamamatsu, Japan).

Results: Placental TOI values in pregnant women with pregnancy-induced hypertension and intrauterine fetal growth restriction were significantly higher than those of pregnant women without complications, although there were no differences of TOI values between the women with threatened preterm delivery and those without complications.

Conclusion: Non-invasive monitoring of placental tissue oxygenation assessed by NIRS for the study of utero-placental function may have a high potential for clinical application, particularly in prenatal management.
\end{abstract}

Keywords: Near-infrared spectroscopy, placenta, oxygenation, tissue oxygenation index (TOI), IUGR.

\section{INTRODUCTION}

Fetal heart rate (FHR) monitoring is widely applied to evaluate the fetal well-being. Long-term follow-up studies on intrapartum FHR monitoring have shown little or no benefit regarding neonatal outcomes but have demonstrated significant improvements in operative deliveries [1,2]. Furthermore, it has been reported that fetal hypoxia cannot be detected accurately by FHR monitoring alone. Therefore, there is a clear need for the development of techniques beyond FHR monitoring for fetal assessment.

The pulse oxymeter is capable of determining the degree of oxygen saturation non-invasively and continuously, by measuring pulsating wave absorbance in peripheral tissues, such as the fingertips. van Oudgaarden et al. [3] reported that oxygen dynamics could be measured over the course of delivery using a pulse oximetry probe applied trans-vaginally to the fetal head. The method is, however, essentially an invasive procedure, as the probe is inserted into the vagina only after the rupture of membrane. Although perinatologists have focused their attention on establishing a method for the early detection of fetal hypoxia to prevent hypoxic damage, no currently established non-invasive methods exist for detecting fetal hypoxia.

*Address correspondence to this author at the Department of Obstetrics and Gynecology, Hamamatsu University School of Medicine, Hamamatsu, Japan; Tel: +81-453-435-2309; Fax: +81-453-435-2308;

E-mail: kanayama@hama-med.ac.jp
A successful pregnancy is dependent upon adequate placental perfusion via the maternal and fetal circulation. Specifically, if the placenta becomes hypoxic, the fetus is exposed to a hypoxic state that can result in serious complications, such as cerebral palsy and neurologic sequelae [4,5]. Thus, evaluating the oxygenation status of the placenta can be critical for determining fetal status. Currently, only antenatal methods for evaluating placental function, which involve diagnostic imaging by ultrasound examination, are available, whereas no direct methods have been established to determine the functional status of the placenta. The ability to measure placental oxygenation during the intrapartum period would provide valuable information about the oxygenation of the fetus.

Near-infrared spectroscopy (NIRS), which was first described by Jobsis in 1977 [6], is a noninvasive and convenient technique for monitoring the regional tissue oxygenation of a number of intact organs [7,8]. This technique is reliant upon the transparency of biological tissues to illuminate tissue chromophores, such as hemoglobin and cytochrome Aa3, in the infrared region of the spectrum. As the absorption of near-infrared light depends on the oxygenation state, a change in chromophore concentration can be quantified using the modified Lambert-Beer law. This technique enables the continuous evaluation of tissue oxy-hemoglobin $(\mathrm{HbO} 2)$ and hemoglobin $(\mathrm{Hb})$ concentrations and allows trained individuals to perform non-invasive monitoring of oxygen dynamics in living bodies. 
For near-infrared light rays, light-scattering events in tissues are approximately two orders of magnitude greater than those related to absorption. This allows near-infrared light to penetrate several centimeters into a tissue before being absorbed or emitted though the boundaries of the tissue and enables the sampling of large tissue volumes. Furthermore, the emitted photons undergo random-walk patterns as they propagate through the tissue, and the resulting photon distribution patterns exhibit a variety of paths in the underlying tissue volume between the near-infrared light source and the detector. Maximal photon distribution occurs at a depth that is approximately one-half of the distance between the source and detector.

The distance between the abdominal surface and the placenta is approximately 2 to $3 \mathrm{~cm}$. Therefore, near-infrared light can penetrate through the placenta if the placenta is located at the anterior wall of the uterus. It was speculated that if we could measure the oxygen concentration of the placenta, we could provide a new method for intrapartum monitoring. In 2005, we reported the application of transabdominal NIRS to the human placenta and successfully evaluated concentrations of $\mathrm{HbO} 2$ and $\mathrm{Hb}$ non-invasively [9]. Subsequently, we proposed the placental tissue oxygen index (TOI) as an index for the assessment of placental oxygenation [10-12].

In the current study, we demonstrated this evaluation of placental function by NIRS, including the application of NIRS for the non-invasive estimation of placental tissue oxygenation and utero-placental function and the assessment of placental oxygenation by TOI. The potential clinical applications of near-infrared spectroscopic quantitative measurements of placental tissue oxygenation for the noninvasive estimation of utero-placental function are also discussed.

\section{METHODS}

\section{Measurement of Placental Oxygenation Using Near- Infrared Spectroscopy}

A method was developed for the trans-abdominal measurement of placental oxygenation using NIRS at the Hamamatsu University School of Medicine Hospital.

As near-infrared lights are highly permeable through living tissue, trans-abdominal NIRS was conducted with NIRO instruments, such as the NIRO-300 and NIRO-100 models (Hamamatsu Photonics; Hamamatsu, Japan), which were more recent generations of near-infrared spectrometers, and permitted additional measurements of the quantitative tissue oxygenation index (TOI), representing the ratio of $\mathrm{HbO} 2$ to total hemoglobin $(\mathrm{HbO} 2 /(\mathrm{HbO} 2+\mathrm{Hb}) \times 100)$. In contrast to the modified Lambert-Beer law, the underlying technical principal of the TOI measurement, spatially resolved spectroscopy (SRS), permits oxygenation measurements in homogenous tissue even when the tissue path length factor is unknown $[13,14]$.

A NIRS probe (Hamamatsu Photonics; Hamamatsu, Japan) consists of a near-infrared light emitter optode and a receiver optode. The NIRS probe was linked to a precalibrated measuring unit, which was connected to the NIRO measurement display unit (Hamamatsu Photonics). Using the

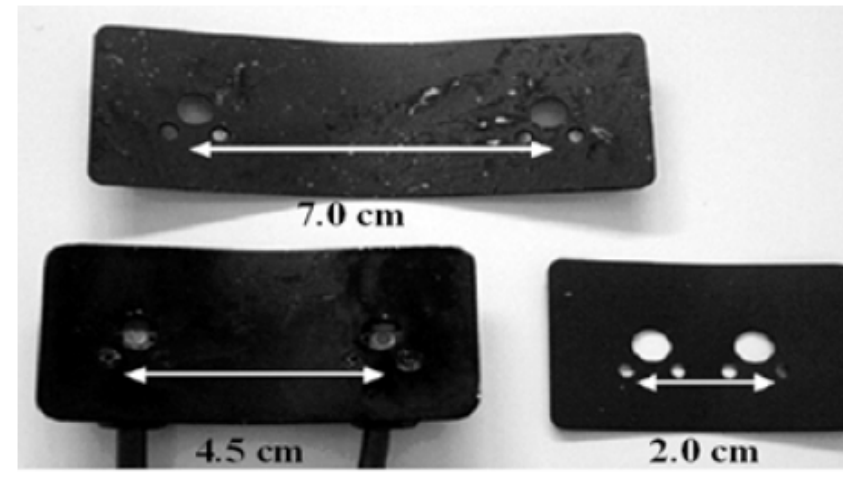

Fig. (1). Three NIRS probes were developed, for which the distance between the near-infrared light source and the detector was $2.0 \mathrm{~cm}$, $4.5 \mathrm{~cm}$ and $7.0 \mathrm{~cm}$.

NIRO measurement, the sample is irradiated with four light beams of different wavelengths $(775,810,847$ and $919 \mathrm{~nm})$ via fiber optics, and the transmitted light is detected using a photomultiplier tube. The detected light is analyzed for the absorption spectrum, and the changes in $\mathrm{Hb}$ and $\mathrm{HbO} 2$ concentrations. This setup enables the calculation of an absolute value for the TOI. The safety of the NIRO measurement apparatus has been well established; the optical density on the skin is approximately $25 \mathrm{~mW} / \mathrm{cm} 2$, which is well below the allowed value of $200 \mathrm{~mW} / \mathrm{cm} 2$. NIRO measurements have been approved for this use by the Japanese government.

\section{Determination of Appropriate Inter-Optode Distance in the NIRS Probe}

The distance between the emitter optode and the detector optode is important. If the distance is too short, the light will propagate through the superficial maternal abdomen. Prior to determining an appropriate inter-optode distance, ultrasound examinations were performed on 31 pregnant women to determine the average distance between the abdominal surface and the internal uterine wall. The mean distance between the skin surface and the internal uterine wall in these pregnant women was $1.9 \pm 0.3 \mathrm{~cm}(\mathrm{SD})$.

Computer simulations performed prior to the present study showed that near-infrared light would penetrate a distance equivalent to the distance from the base to the apex of an equilateral triangle if the source-detector separation was equal to the length of the base of the triangle [15]. We developed three probes for which the distance between the near-infrared light source and the detector was $2.5 \mathrm{~cm}, 4.5$ $\mathrm{cm}$ and $7.0 \mathrm{~cm}$ (Fig. 1). Using these probes, trans-abdominal NIR measurements were performed on pregnant women whose placentas adhered to the anterior wall of the uterus to identify a sufficient distance for the detection of nearinfrared light propagated to the placenta. These probes were attached to the maternal skin surface of each participant at a position just above the placenta.

For each of participants, it was possible to determine concentrations of $\mathrm{HbO} 2$ and $\mathrm{Hb}$ with the probe of which inter-optode distance was set at $4.5 \mathrm{~cm}$. Although fetal movements were observed by ultrasound during the period of measurement, the concentrations of $\mathrm{HbO} 2$ and $\mathrm{Hb}$ were stable at fetal movements. It was, however, impossible to determine concentrations of $\mathrm{HbO} 2$ and $\mathrm{Hb}$ when the inter- 
(a)

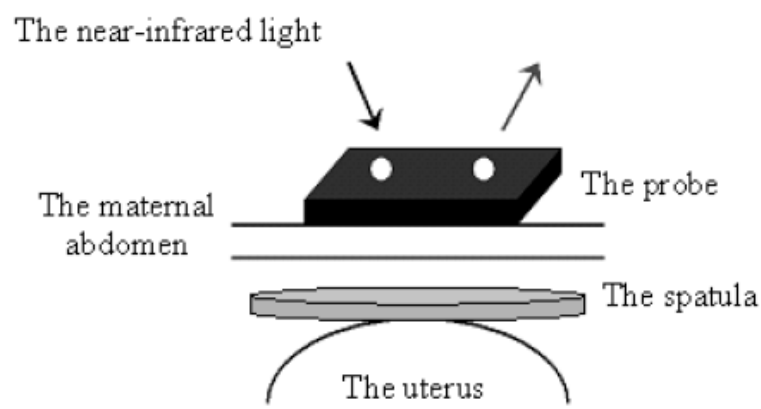

(b)

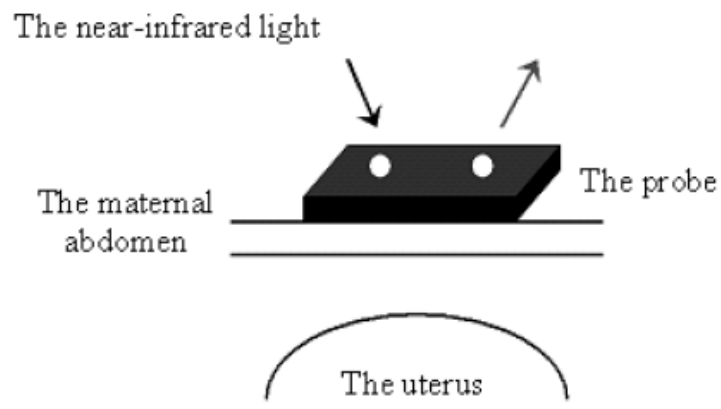

Fig. (2). The reach of near-infrared light to the placenta was confirmed by the $\mathrm{HbO} 2$ and $\mathrm{Hb}$ levels of myometrium during elective cesarean section. (a) The probe was attached the maternal surface and the oxygenation level was measured after placing a spatula on the uterine surface. (b) Myometrial tissue oxygenation level was measured without the spatula.

optode distance of NIRS probe was set at 2.0 or $7.0 \mathrm{~cm}$. Therefore, the distance between emitter and detector optodes was fixed at $4.5 \mathrm{~cm}$ in the NIRS probe holder in the present study.

\section{Confirmation of the Distance Reached by Near-Infrared Light}

Four experiments were performed to confirm the ability of near-infrared light to reach the placenta $[9,10]$. Each experiment was performed after the informed consent of participants.

The NIRS probe was attached to the maternal skin surface just at the placental position identified by ultrasound image, and a total of 45 trans-abdominal measurements were performed by NIRS in 11 pregnant women. In five of the 11 participants, the NIRS measurements were repeated after delivery at the same position that was used for the antenatal measurement. The mean $\mathrm{HbO} 2 / \mathrm{Hb}$ ratio $\pm \mathrm{SD}$ was $2.49 \pm$ 0.61 in women during pregnancy and was $4.83 \pm 1.25$ after delivery [9].

$\mathrm{HbO} 2$ and $\mathrm{Hb}$ levels were measured in the myometrium by NIRS during cesarean section in two cases. The probe was attached to the maternal skin surface and measured the tissue oxygenation levels immediately prior to closing the incision with and without placing a spatula between the uterus and abdominal wall (Fig. 2). The spatula was made of metal and did not allow the penetration of near-infrared light. $\mathrm{As} \mathrm{HbO} 2$ and $\mathrm{Hb}$ levels decreased when the spatula was placed on the uterus, the NIRS method was able to detect and quantify the near-infrared light that had reached the uterus [10].

The influence of tissue specificity on the NIRS measurements was studied, as there are the placenta, fetus and amniotic fluid in the pregnant uterus and the trans-abdominal NIRS measurements may alter according to the subject. Actually, the mean $\mathrm{TOI} \pm \mathrm{SD}$ values were significantly changed among the subjects, and they were $70 \pm 3.9 \%, 80 \pm 2.4 \%$ and $54 \pm 3.9 \%$ in the placenta, fetus and amniotic fluid, respectively [10]. Therefore, the subject was studied with ultrasound imaging in the trans-abdominal NIRS to confirm whether placental oxygenation was measured.

The TOI values of the placenta were measured after delivery to determine whether the TOI value reflects the oxygenation in the antenatal placenta. The NIRS probe was attached to the maternal side of placenta immediately after the placenta was expelled, then repeatedly measured the TOI values. It was approximately $70 \%$ immediately after the birth, which was similar to those measured during pregnancy, decreased steadily and reduced to $40 \% 5$ minutes after the delivery [10].

\section{RESULTS}

\section{Clinical Evaluation of Placental Oxygenation by Trans- Abdominal NIRS}

After NIRS method was introduced for the transabdominal measurement of placental oxygenation, its clinical utility was evaluated for the non-invasive estimation of utero-placental function during antepartum period [10-12].

Each utility was evaluated in pregnant women who were managed at the Hamamatsu University School of Medicine Hospital. All pregnant women who were eligible for the evaluation underwent an ultrasound examination prior to the NIRS studies to identify the placental location. Women who had posteriorly located placentas were excluded because scattering and absorption could have affected the detection of changes in the near-infrared light signals. Women with multiple pregnancies were also excluded. Each evaluation was performed after informed consent was obtained from the women.

The NIRS probe with an inter-optode distance of $4.5 \mathrm{~cm}$ (Hamamatsu Photonics;Hamamatsu, Japan) was attached to the maternal skin surface of each participant at the position just above the placenta, and TOI values were recorded by trans-abdominal NIRS. Interference from external light was prevented by shielding, and the TOI values were continuously measured and recorded. The results were recorded and stored as numerical values in a personal computer. The TOI was calculated as the median value, and the sampling time was set at 10 minutes.

\section{TOI Values in Pregnant women Without Complications}

A total of 79 measurements of trans-abdominal NIRS were performed at 25 to 40 weeks of gestation on 15 pregnant women without complications, and the mean TOI \pm SD 
value was $72.3 \pm 5.3 \%$. There were no differences in TOI values related to the gestational age at the measurement [11, 12].

\section{TOI Values in Pregnant Women with Complications}

TOI values of pregnant women without complications were compared with those in women with pregnancyinduced hypertension (PIH) [11], threatened preterm delivery (TPD) [12] and intrauterine fetal growth restriction (FGR) [10].

The placental function was assessed by trans-abdominal NIRS during pregnancy in 6 women with PIH and 15 pregnant women without complications. The mean TOI \pm SD value was significantly higher in women with PIH than in pregnant women without complications $(80.2 \pm 2.7 \%$ vs. $72.3 \pm 5.3 \%$ ). There was no difference in the mean TOI \pm SD values between the women with mild and severe PIH $(80.1 \pm 3.4 \%$ vs. $80.5 \pm 2.8 \%)$ [11]. TOI values in 15 pregnant women with FGR $(78.6 \pm 1.6 \%)$ were significantly higher than those of 15 pregnant women without complications $(70.2 \pm 0.4 \%)$ by using trans-abdominal NIRS [10].

TOI values in 6 women with TPD, who had been admitted with intact membranes, were compared to those of 15 pregnant women without complications. There was no differences in the mean TOI \pm SD values between women with TPD and those without complications $(72.9 \pm 5.1 \%$ vs. 72.3 $\pm 5.3 \%$ ) using trans-abdominal NIRS. The TOI value at the admission was compared to that after effective tocolysis with beta-agonist infusion began at the admission and lasted for $11.8 \pm 3.9$ days. There was no difference in the TOI values before and after the tocolytic treatment [12].

\section{DISCUSSION}

For women with utero-placental dysfunction, placental oxygenation should be evaluated and an intervention to promote placental blood flow and oxygen delivery should be prioritized. The currently available approaches for evaluating placental function rely on diagnostic imaging such as ultrasound examination and/or the fetal or umbilical cord blood flow velocity studied by pulsed Doppler. It is important to perform physiological assessments of the oxygen dynamics in the placenta. No methods, however, currently exist for determining the oxygenation status of the placenta during pregnancy. Thus, a method to monitor placental tissue oxygenation was developed in this study by trans-abdominal NIRS and evaluated the feasibility of TOI values measured using NIRS.As described herein, we successfully measured TOI values in pregnant women and found that the values were greater in pregnant women with PIH and FGR than in pregnant women without complications. As placental hypoxia is reflective of the fetus oxygen status, this new method is expected to contribute to improvements in perinatal management.

The inter-optode distance was set to $4.5 \mathrm{~cm}$ to monitor placental tissue oxygenation in the probe of trans-abdominal NIRS. Ramanujam et al. [16] reported that for the transabdominal NIRS measurements of fetal cerebral blood oxygenation, near-infrared light was expected to migrate through both the underlying maternal and the fetal tissue before its detection at abdominal surface when a probe of which sepa- ration distance was $10.0 \mathrm{~cm}$ between the source and the detector [13]. We confirmed that near-infrared light reached the placenta through four experiments, i.e. firstly the difference in the mean $\mathrm{HbO} 2$ to $\mathrm{Hb}$ ratio during pregnancy and after delivery [9] suggested that the data measured after delivery indicated the oxygenation status of the maternal abdomen alone whereas those data measured during pregnancy indicated the status of both the maternal abdomen and placenta. Secondly, the near-infrared light values changed when a metal spatula was placed between the uterus and abdominal wall, which suggested that near-infrared light did not reach the uterus when it was blocked by the spatula [10]. The result will indicate that this method is capable to detect the near-infrared light that reached the uterus.

Thirdly, remarkable changes were detected in the TOI values when different subjects were measured, such as the placenta, fetus and amniotic fluid, which suggested that the near-infrared light reached inside of the uterus and distinguished tissue differences [10]. Forth, we observed decreasing TOI values in the expelled placenta immediately after delivery [10], i.e. TOI values obtained by NIRS will be an appropriate indicator of placental oxygenation. Thus, penetration depth of the near-infrared light would be sufficient to propagate light through the placental tissue.

Although it is uneasy to measure fetal oxygen dynamics noninvasively due to fetal movement, the placenta dose not move and enables the measurement of stable placental TOI values.

Considering the experimental results, TOI values was evaluated in the placenta by trans-abdominal NIRS in the antepartum period. The TOI values were calculated from the detection of oxyhemoglobin and deoxyhemoglobin by the exposure to near-infrared light. The maternal blood in the intervillous spaces and the fetal blood in the chorionic villous vasculature circulate separately but they spread in the placental tissue. Since the intervillous spaces are wider than those of villus capillaries in placental histology, it is strongly suggested that oxyhemoglobin and deoxyhemoglobin amounts in the intervillous spaces are much greater than those in the villous capillaries, as assessed by NIRS. Therefore, it is plausible that the TOI value mainly reflects the oxygenation of the intervillous blood.

In our study, TOI values measured by the NIRS in noncomplicated pregnant women did not differ in regard to gestational age and indicated the effective transport of oxygen from the maternal blood to the fetus in the placenta [11]. It was also demonstrated that the mean TOI values in women with PIH and those with FGR were significantly higher than the values in non-complicated pregnant women [10,11]. Kaufmann et al. [17] suggested that preeclampsia is associated with the generalized impairment of trophoblast invasion. Prefumo et al. [18] also suggested that the poor development of the placenta in early pregnancy may cause preeclampsia. Although the etiology of FGR is controversial, reduced fetal growth can be ascribed to placental insufficiency. Xiong et al. [19] noted that neonates with placental dysfunction often exhibit asymmetric body proportions and reduced overall growth. Placental oxygenation could be affected in cases of FGR and may lead to decreased perfusion, which could further affect the vessel stream of the chorionic 
plate [20]. The larger TOI values obtained in our study suggest that PIH and FGR may be caused by problems related to utero-placental dysfunction. It is speculated that oxygen transfer from maternal to fetal blood may be impaired due to dysfunctional villi, and as a result, there would be an increased oxygen concentration in the intervillous spaces that would yield greater TOI values. As measured by NIRS, TOI values may be an indicator of villous or placental function.

Ogino and Redline [21] postulated that the pathogenesis of chorangiosis and hypercapillarization was related to an increased intramural pressure, which may be related to venous obstruction at the umbilical cord or the fetal cardiac level. Suzuki et al. [22] reported that placental TOI values were significantly elevated in cases of chorangiosis, and these authors suggested that greater TOI values in the intervillous spaces may precede low-efficiency gas exchange in the villous membranes in cases of placentas with chorangiosis. Although no decreased TOI values were observed in our studies, Hasegawa et al. [23] reported that TOI values from cases with small for gestational age (SGA) with severe pre-eclampsia and placental abnormality were significantly higher than in appropriate for gestational age (AGA), while TOI values in the SGA with umbilical cord abnormalities were lower than in AGA. The umbilical cord abnormalities may promote the oxygen perfusion into terminal villi from the intervillous space supposedly due to villous complications, then lower the oxygen and oxygenated hemoglobin in the intervillous space.

Unlike conventional methods, this NIRS system invented for the trans-abdominal measurement of placental dynamic oxygenation during the antepartum period is a non-invasive and simple technique. However, the main complications with this system were the inability to compare the TOI value to a placental blood sample and the restriction of the measurement site to a small portion of the placenta. Furthermore, posteriorly located placentas were not evaluated because scattering and absorption would have affected the detection of the change of near-infrared light signals.

Further clinical investigations are required to evaluate the clinical efficacy of this method for the assessment of placental function. Many factors can affect placental oxygenation, and only four types of cases were investigated in this study: pregnancies without complications, those with PIH, FGR and TPD. Further clinical investigations are required to reduce the risk of bias and to evaluate the potential value of this technique in clinical practice.

Despite the limitations of TOI values measured using NIRS, the potential to provide a non-invasive monitoring technique for placental oxygenation is a valuable concept. Furthermore, the evaluation of TOI values has the potential to identify placental dysfunction earlier during pregnancy. As placental dysfunction can result in intrapartum fetal hypoxia as well as neonatal complications, we propose that transabdominal NIRS should be considered as a candidate of non-invasive monitoring technique for measuring placental oxygenation on a real-time basis during the antepartum period.

\section{CONCLUSIONS}

The development of a novel, non-invasive monitoring technique was developed for measuring placental oxygenation by trans-abdominal NIRS and TOI values were evaluated using this system in the present study. This novel monitoring technique has a large potential to be used as a noninvasive measurement that is both safe and simple to apply, particularly in prenatal management.

\section{CONFLICT OF INTEREST}

The authors declare no conflict of interests.

\section{ACKNOWLEDGEMENTS}

We thank Mr. Hideki Maeda of Hamamatsu Photonics K.K. for his technical advice.

\section{REFERENCES}

[1] Murphy KW, Johnson P, Moorcraft J, Pattinson R, Russell V, Turnbull A. Birth asphyxia and the intrapartum cardiotocograph. Br J Obstet Gynecol 1990; 97(6): 470-9.

[2] Nelson KB, Dambrosia JM, Ting TY, Grether JK. Uncertain value of electronic fetal monitoring in predicting cerebral palsy. $\mathrm{N}$ Engl $\mathrm{J}$ Med 1996; 334(10): 613-8.

[3] van Oudgaarden E, Johnson N. Clinical value of antenatal fetal pulse oximetry. J Perinat Med 1994; 22(4): 295-300.

[4] Mallard EC, Williams CE, Johnston BM, Gluckman PD. Neuronal damage in the developing brain following intrauterine asphyxia. Reprod Fertil Dev 1995; 7(3): 647-53.

[5] Rivkin MJ. Hypoxic-ischemic brain injury in the term newborn. Neuropathology, clinical aspects, and neuroimaging. Clin Perinatol 1997; 24(3): 607-25.

[6] Jobsis FF. Noninvasive infrared monitoring of cerebral and myocardial oxygen sufficiency and circulatory parameters. Science 1977; 198: 1264-7.

[7] Pennekamp CW, Bots ML, Kappelle LJ, Moll FL, de Borst GJ. The value of near-infrared spectroscopy measured cerebral oximetry during carotid endarterectomy in perioperative stroke prevention. A review. Eur J Vasc Endovasc Surg 2009; 38(5): 539-45.

[8] Shadgan B, Guenette JA, Sheel AW, Reid WD. Sternocleidomastoid muscle deoxygenation in response to incremental inspiratory threshold loading measured by near infrared spectroscopy. Respir Physiol Neurobiol 2011; 178(2): 202-9.

[9] Kakogawa J, Sumimoto K, Ho E, Kanayama N. Transabdominal measurement of oxygenation of the placenta by near-infrared spectroscopy. Semin Thromb Hemost 2005; 31(3): 297-301.

[10] Kawamura T, Kakogawa J, Takeuchi Y, et al. Measurement of placental oxygenation by transabdominal near-infrared spectroscopy. Am J Perinatol 2007; 24(3): 161-6.

[11] Kakogawa J, Sumimoto K, Kawamura T, Minoura S, Kanayama N. Transabdominal measurement of placental oxygenation by nearinfrared spectroscopy. Am J Perinatol 2010; 27(1): 25-9.

[12] Kakogawa J, Sumimoto K, Kawamura T, Minoura S, Kanayama N. Noninvasive monitoring of placental oxygenation by near-infrared spectroscopy. Am J Perinatol 2010; 27(6): 463-8.

[13] Matcher SJ, Kirkpatric P, Nahid K, Cpe M, Delpy DT. Absolute quantification methods in tissue near infrared spectroscopy. Proc SPIE 1995; 2389: 486-95.

[14] Suzuki S, Takasaki S, Ozaki T, Kobayashi T. A tissue oxygenation monitor using NIR spatially resolved spectroscopy. Proc SPIE 1999; 3579: 144-5.

[15] Elwell CE. A Practical Users Guide to NIRS. Hamamatsu, Photonics KK: Japan 1995; pp. 38-41.

[16] Ramanujam N. Photon migration though fetal head in utero using continous wave, near infrared spectroscopy: clinical and experimental model studies. J Biomed Opt 2000;5:173-84.

[17] Kaufmann P, Black S, Huppertz B. Endovascular trophoblast invasion: implications for the pathogenesis of intrauterine growth retardation and preeclampsia. Biol Reprod 2003; 69(1): 1-7. 
[18] Prefumo F, Sebire NJ, Thilaganathan B. Decreased endovascular trophoblast invasion in first trimester pregnancies with highresistance uterine artery Doppler indices. Hum Reprod 2004; 19(1): 206-9.

[19] Xiong X, Demianczuk NN, Buekens P, Saunders LD. Association of preeclampsia with high weight for gestational age. Am J Obstet Gynecol 2000; 183(1): 148-55.

[20] Kingdom JC, Kaufmann P. Oxygen and placental villous development: origins of fetal hypoxia. Placenta 1997; 18(1): 613-21.
[21] Ogino S, Redline RW. Villous capillary lesions of the placenta: distinctions between chorangioma, chorangiomatosis, and chorangiosis. Hum Pathol 2000; 31(8): 945-54.

[22] Suzuki K, Itoh H, Kimura S, et al. Chorangiosis and placental oxygenation. Congenit Anom 2009; 49(2): 71-6.

[23] Hasegawa J, Nakamura M, Matsuoka R, et al. Evaluation of placental function using near infrared spectroscopy during fetal growth restriction. J Perinat Med 2010; 38(1): 29-32.

Received: January 14, 2012

Revised: March 262012

Accepted: March 28, 2012

(C) Kakogawa and Kanayama; Licensee Bentham Open.

This is an open access article licensed under the terms of the Creative Commons Attribution Non-Commercial License (http://creativecommons.org/licenses/by-nc/3.0/) which permits unrestricted, non-commercial use, distribution and reproduction in any medium, provided the work is properly cited. 